Canadian Journal of Applied Linguistics

Revue canadienne de linguistique appliquée

\title{
L'usage de l'anglais dans une école de langue française en milieu minoritaire : un véritable enjeu ?
}

\section{Callie Mady}

Volume 22, Number 2, 2019

URI: https://id.erudit.org/iderudit/1063774ar

DOI: https://doi.org/10.7202/1063774ar

\section{See table of contents}

Publisher(s)

University of New Brunswick

ISSN

1920-1818 (digital)

Explore this journal

Cite this article

Mady, C. (2019). L’usage de l'anglais dans une école de langue française en milieu minoritaire : un véritable enjeu ? Canadian Journal of Applied Linguistics / Revue canadienne de linguistique appliquée, 22(2), 64-87.

https://doi.org/10.7202/1063774ar

\section{Article abstract}

This article presents the results of a study whose goal was to support French language learners and their teachers, in kindergarten classes in a French language school in Ontario. Data were collected through classroom observations and interviews with the teachers and early childhood educators, who also participated in a professional learning community addressing the needs of French language learners. The participants revealed that language use was their biggest concern. The majority of these teachers and early childhood educators indicated that participation in the professional learning community influenced their use of English in class. The participants stated that they had increased consciousness and confidence in students' ability to learn French without the use of English, which motivated them to progressively reduce their English use while encouraging students' French use.
Copyright (c) Callie Mady, 2019

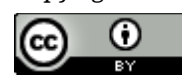

This document is protected by copyright law. Use of the services of Érudit (including reproduction) is subject to its terms and conditions, which can be viewed online.

https://apropos.erudit.org/en/users/policy-on-use/ 


\title{
L'usage de l'anglais dans une école de langue française en milieu minoritaire : un véritable enjeu ?
}

\author{
Callie Mady \\ Nipissing University
}

\begin{abstract}
Résumé
Cet article présente les résultats de notre étude dont le but était d'appuyer les apprenants de français inscrits au Programme d'apprentissage des jeunes enfants (PAJE) et de soutenir les enseignants d'une école de langue française en Ontario. Nous avons recueilli des données grâce à des observations en classe et des entrevues avec les enseignantes et éducatrices. Nous avons aussi participé aux réunions de la communauté d'apprentissage au sujet des apprenants. Les enseignantes et les éducatrices participantes ont indiqué que la langue de communication était le plus grand enjeu auquel elles faisaient face. Selon la majorité des participantes, les réunions ont influencé leur usage de l'anglais en salle de classe. Les participantes ont affirmé avoir une meilleure conscience et une confiance accrue dans la capacité de leurs élèves d'apprendre le français sans utiliser l'anglais, ce qui les encourageait à réduire progressivement l'utilisation de l'anglais et les motivait aussi à inciter les élèves à utiliser davantage le français.
\end{abstract}

\begin{abstract}
This article presents the results of a study whose goal was to support French language learners and their teachers, in kindergarten classes in a French language school in Ontario. Data were collected through classroom observations and interviews with the teachers and early childhood educators, who also participated in a professional learning community addressing the needs of French language learners. The participants revealed that language use was their biggest concern. The majority of these teachers and early childhood educators indicated that participation in the professional learning community influenced their use of English in class. The participants stated that they had increased consciousness and confidence in students' ability to learn French without the use of English, which motivated them to progressively reduce their English use while encouraging students' French use.
\end{abstract}




\section{L'usage de l'anglais dans une école de langue française en milieu minoritaire : un véritable enjeu?}

\section{Contexte de l'étude}

Au Canada, le français et l'anglais sont les deux langues officielles du pays (Gouvernement du Canada, 1969). Par conséquent, les citoyens ont le droit de faire instruire leurs enfants dans la langue de la minorité. En Ontario, où le français est la langue de la minorité, selon l'article 23 de la Charte canadienne des droits et libertés (Gouvernement du Canada, 1982), les personnes considérées comme des ayants droit et peuvent alors faire instruire leurs enfants ont français sont celles qui (a) ont appris le français comme première langue et le comprennent encore ou (b) ont fréquenté une école primaire de langue française au Canada ou (c) ont un enfant qui a reçu ou reçoit son éducation primaire ou secondaire en langue française au Canada.

Les conseils scolaires de langue française de l'Ontario accueillent les enfants des ayants droit. Tout autre parent voulant y inscrire son enfant peut faire une demande à un comité d'admission. Dans l'école qui a participé à cette étude, $56 \%$ des parents d'enfants inscrits au Programme d'apprentissage des jeunes enfants (PAJE) ont signalé qu'ils étaient des ayants droit et l'école a identifié $90 \%$ des élèves inscrits au PAJE comme étant des apprenants du français.

D'abord, cette étude avait comme but d'appuyer les enseignants d'une école de langue française et de soutenir les apprenants inscrits au PAJE. Les données ont révélé l'importance de l'enjeu de la langue et, en particulier, de l'usage de l'anglais dans une école de langue française. Cet article cherche à éclairer ces questions de langue à l'aide de données et de recherches.

\section{Revue documentaire}

\section{La politique gouvernementale}

Le ministère de l'Éducation de l'Ontario (2004) a élaboré la Politique de l'aménagement linguistique de l'Ontario $(\mathrm{PAL})$ pour aider les écoles à faire face à la diversité linguistique dans les écoles de langue française. Ce document a reconnu la nécessité d'un aménagement linguistique, en raison du contexte ontarien anglodominant qui pose des défis quant à la transmission de la langue et de la culture françaises. Pour répondre à ce défi, la PAL a suggéré, sans donner de précisions, que les enseignants utilisent une approche pédagogique adaptée au milieu minoritaire et qui tient compte du bagage linguistique varié des élèves.

De plus, le Conseil des ministres de l'Éducation (CMEC ; 2003, 2004) a élaboré des projets collectifs, par exemple le Projet canadien de français langue première. Le but de ce projet pancanadien était, entre autres, d'améliorer l'apprentissage du français des élèves en milieu minoritaire. Ce projet se divisait en trois phases. La première phase avait une double composante, notamment, une recherche portant sur les résultats des élèves qui a révélé un niveau de rendement inférieur des francophones (2004) et une trousse de francisation créée pour les enseignants de la maternelle à la $2^{\mathrm{e}}$ année (2003). La deuxième phase visait la communication orale et les stratégies d'apprentissage. La troisième phase se concentrait sur l'appropriation de la culture. 
La présente étude s'est limitée à la première phase, à la littératie en particulier, en recommandant des interventions qui ciblaient les stratégies d'apprentissage. Le Projet canadien de français language première (CMEC, 2003, 2004) a souligné l'importance d'adopter une approche pédagogique qui répond aux besoins des élèves en milieu minoritaire, mais sans traiter de la question de l'acquisition de la langue. La deuxième composante de la première phase concernait la trousse de francisation qui a développé une approche pédagogique conçue pour répondre aux besoins des élèves en milieu minoritaire et comportait 10 principes indispensables. D'abord, que le personnel enseignant reconnaisse que les élèves sont exposés aux deux langues officielles, qu'il a un mandat de former des élèves francophones bilingues et que l'assimilation est une réalité. Qu'il sache aussi qu'un rapport positif à la langue constitue la base de tout enseignement en milieu francophone minoritaire, qu'un accent sur la culture francophone, une attention à l'image de l'élève en tant que francophone et un esprit ouvert à la francophonie sont incontournables dans ce milieu.

Plus récemment, la Fédération canadienne des enseignantes et des enseignants (FCE) a élaboré une pédagogie de la langue française (FCE, 2014b) qui comporte deux conditions, notamment la prise en compte des relations interpersonnelles et du partage de l'influence. La FCE a recommandé aux enseignants d'utiliser une approche culturelle qui soutient la construction identitaire des élèves et encourage l'appropriation de la culture par l'élève. La pédagogie ne touchait pas à l'apprentissage de la langue, bien qu'elle ait mentionné que la langue était le véhicule de la culture.

\section{L’approche pédagogique selon la recherche}

Au Canada, en plus de tenir compte des recommandations gouvernementales, des chercheurs se sont penchés également sur l'étude des approches pédagogiques pour déterminer laquelle conviendrait le mieux au contexte francophone minoritaire. La majorité ont proposé de recourir à l'approche pédagogique du français langue maternelle (FLM), plutôt qu'à l'approche pédagogique du français langue seconde (FLS). Parmi les raisons invoquées par les recherches (p. ex. Berger, 2007 ; Cormier, 2005) en faveur de l'approche du FLM, il y a la croyance que le français pourrait devenir, par cette occasion, la langue d'usage dominante pour les élèves et devrait être gérée par des francophones (c.-à-d. ceux qui ont appris le français comme langue première).

L'approche pédagogique la plus citée est celle de Cazabon (1997). Selon Cazabon (1997), la dimension langue s'articule autour de trois composantes : linguistique, discursive et pragmatique. La première composante concerne la structure de la langue, la deuxième, l'acte de la communication et la troisième, la situation de communication. L'un des défis majeurs reliés à cette approche pédagogique, dans son adoption, demeure le manque d'attention accordée à la mise en œuvre de stratégies appropriées. Cazabon (1997) reconnait cette lacune dans ses recommandations et suggère aux enseignants de tenter d'y remédier.

De même, Landry (2003) a proposé une pédagogie actualisante et communautarisante comme approche au contexte francophone minoritaire. D'une part, la pédagogie actualisante aide les élèves à découvrir leur unicité et, en même temps, celle de leur collectivité afin de réaliser leur potentiel et de pouvoir jouer leur rôle dans la communauté. D'autre part, la pédagogie communautarisante met de l'avant cinq composantes : l'enculturation active, le développement de l'autodétermination, 
l'actualisation maximale du potentiel d'apprentissage, la conscientisation et l'engagement, et l'entrepreneuriat communautaire. Landry a reconnu toutefois que cette pédagogie offrait des liens directeurs sans offrir de stratégies précises à adopter et ne s'adressait pas à l'apprentissage de la langue de façon explicite.

Dans la même perspective, Cormier (2005) a proposé une approche par ricochet qui met l'accent sur le va-et-vient entre l'élève et la communauté. Pour la mise en œuvre efficace de cette approche, Cormier (2005) a identifié huit éléments essentiels, dont le développement d'un rapport positif à la langue et l'optimalisation de la production et de la réception langagières en français, suivi de l'enculturation active, l'actualisation maximale du potentiel d'apprentissage, le développement de l'autodétermination, la négociation identitaire, et pour terminer, la conscientisation et la littératie critique vers l'engagement, les partenariats communautaires et l'entrepreneuriat communautaire. Cormier (2005) a aussi offert des précisions sur l'application de ces principes en classe. Par exemple, elle a suggéré de faire participer les élèves à des activités communautaires afin de leur offrir des occasions sociales où ils pouvaient augmenter l'usage du français, de les exposer à une variété de registres de français menant au registre scolaire et de valoriser l'oral et les littératies. En plus, elle a proposé de ne pas trop simplifier la langue, mais plutôt de la contextualiser pour augmenter la compréhension et modeler l'usage de stratégies tout en utilisant une approche communicative.

De son côté, Berger (2007) a proposé une approche d'actualisation linguistique en français dans un contexte minoritaire, ce qui permettrait d'augmenter les habiletés de prélecture/lecture. Pour sa part, Lentz (2016) a encouragé les enseignants à utiliser une approche pédagogique qui répondait bien au contexte minoritaire. Bien qu'il n'ait pas suggéré d'approche précise, Lentz (2016) a recommandé de mettre en place des situations authentiques, des pratiques de langue variées, un climat sécurisant et une évaluation de l'apprentissage et au service de l'apprentissage.

D'autres chercheurs ont suggéré que des approches pédagogiques mises au point dans d'autres contextes pouvaient s'appliquer en milieu minoritaire. C'est le cas de Masny (2003) qui a proposé l'adoption d'une approche pédagogique des littératies multiples pour la communauté scolaire de langue française minoritaire. Bours et Lentz (2009) ont recommandé également cette approche pédagogique en raison du lien à la langue et du partenariat qu'elle suscite entre l'école et la communauté par le biais de projets authentiques. Bours et Lentz et Masny ont offert des exemples de littératies multiples, mais n'ont donné aucune précision sur le processus, ni sur les adaptations nécessaires qu'il faudrait apporter pour les élèves qui parlent peu la langue.

Enfin, Cormier et Lowe (2010) ont reconnu que l'apprentissage du FLS pourrait servir en tant qu'approche en contexte minoritaire si on y apportait les nuances nécessaires. Cependant, elles n'ont pas relevé les stratégies (p. ex. les gestes, la répétition de structures de phrase) qui rendraient cette approche efficace.

En résumé, la recherche offre des recommandations et des suggestions d'approches pédagogiques pour le milieu minoritaire. La plupart soulignent l'importance d'engager la communauté dans l'apprentissage linguistique des élèves. En plus de cet engagement communautaire, d'autres recommandations faisant consensus insistent sur l'importance d'y incorporer la culture francophone, de recourir aux situations de communication authentiques, d'utiliser une approche communicative et de mettre l'accent sur les habiletés de littératie des élèves. Parmi toutes ces recommandations, Cormier et Lowe (2010) sont les 
seules à reconnaitre que beaucoup d'élèves apprennent le français pour la première fois à l'école et qu'il faut une pédagogie qui en tient compte.

\section{Stratégies de francisation dans les documents gouvernementaux à l'intention du personnel enseignant}

Selon Bell (2007), les enseignants auraient besoin de stratégies précises pour opérationnaliser ces approches en classe. Nous avons donc exploré deux types de documents gouvernementaux pour dégager des stratégies pratiques susceptibles d'être mises en place par les enseignants pour appuyer les élèves qui ne parlent pas le français ou qui le parlent peu.

Dans le but de soutenir le PAJE, le ministère de l'Éducation de l'Ontario (2016) a publié un document intitulé Programme de la maternelle et du jardin d'enfants. Ce document, qui reconnait la présence d'élèves apprenant le français, guide les écoles dans leur appui et leur accompagnement des enfants apprenant la langue afin de répondre à leurs besoins. Le document donne aux écoles la liberté de décider comment répondre au mieux à ces besoins.

La première phase du Projet canadien de français langue première (CMEC, 2003) offre aussi une section sur les pratiques pédagogiques. En ce qui concerne la communication enseignant/élève, il est recommandé dans la trousse de fournir aux élèves certaines structures de phrases simples et le vocabulaire nécessaire pour communiquer et participer aux activités en classe. Le document recommande également d'accompagner les structures de phrase par des gestes, de paraphraser, d'inviter l'élève à paraphraser, de fournir un modèle, de verbaliser le vécu de l'élève, de lui fournir une correction et de reformuler son énoncé. Enfin, pour favoriser l'usage du français par les élèves, les enseignants peuvent demander aux élèves de répéter, peuvent compléter une structure de phrase en fournissant la structure sans un mot ou peuvent fournir en français la phrase dite en anglais par l'élève.

La section du document sur les expériences langagières requiert des situations d'apprentissage qui permettent aux élèves d'acquérir et d'utiliser du vocabulaire. Selon le document, pour faciliter l'apprentissage de nouveau vocabulaire par les élèves en francisation, on doit leur fournir des occasions de jouer avec les mots, de les voir, de les entendre et de les vivre pour se les approprier (CMEC, 2003).

\section{Stratégies de francisation dans la recherche à l'intention du personnel enseignant}

En plus de l'étude de documents gouvernementaux, nous avons également parcouru les articles scientifiques à la recherche de stratégies pratiques que les enseignants pourraient utiliser avec des apprenants de français en milieu minoritaire. Cormier (2011) a suggéré d'encourager les élèves à parler sans trop les corriger et de leur fournir des occasions d'entendre des locuteurs de la langue qui utilisent un registre scolaire. Elle (2011) a aussi proposé de mettre l'accent sur l'enrichissement du vocabulaire à l'aide de jeux de mots, par exemple, et d'utiliser d'autres stratégies de vocabulaire qui pourraient aider à établir un rapport positif au français. Finalement, Cormier (2011) a encouragé l'usage du français comme outil de communication par les élèves dans la communauté pour que la langue ne demeure pas associée uniquement au milieu scolaire. 
MacWillie et LeBlanc (2010), quant à elles, ont fait une distinction entre l'acquisition d'une langue et l'apprentissage du FLM et du FLS. Elles favorisent une approche qui se base sur les points communs entre les deux et s'applique au moyen de jeux et d'activités dramatiques. Pour y parvenir, MacWillie et LeBlanc préconisent certaines stratégies pratiques que les enseignants peuvent utiliser en contexte de francisation : ralentir le débit du langage, simplifier le vocabulaire, utiliser des aides visuelles, adopter une syntaxe plus simple et fournir une variété de techniques de questionnement, dont les questions oui/non, choix multiples et d'association.

Bien que des approches et des stratégies soient suggérées dans les documents gouvernementaux et les articles scientifiques, leur succès n'a pas encore été démontré en milieu minoritaire. Cependant, nous avons trouvé deux études empiriques. Maltais (2007) a mené une étude qui a comparé les résultats de deux groupes d'élèves inscrits à un programme de maternelle : un groupe qui avait le français comme langue principale parlée à la maison et l'autre qui ne l'avait pas. Maltais a comparé les résultats des épreuves de langue et de lecture de ces deux groupes à la fin de la maternelle et à la fin de la $2^{\mathrm{e}}$ année. Elle a trouvé que le programme de maternelle à temps plein avait permis aux élèves qui parlaient peu la langue en dehors de l'école d'atteindre le même niveau que l'autre groupe en ce qui concerne le langage réceptif et la qualité de la lecture, mais pas en compréhension de lecture. Malheureusement, Maltais n'a pas offert de description détaillée de la pédagogie utilisée, ce qui ne permet pas aux enseignants de pouvoir l'adopter.

Par le biais d'un sondage effectué auprès des enseignants de l'actualisation linguistique du français en Ontario, Berger (2003) a exploré les pratiques pédagogiques des enseignants travaillant en majorité avec des enfants du jardin et de la maternelle. Les enseignants ont souligné l'importance de la communication orale et de la diversification des stratégies dans leurs pratiques pédagogiques. Plus précisément, ils ont nommé les techniques de questionnement comme moyen de poser des questions, mais aussi comme stratégie pour faire apprendre aux élèves comment poser des questions. En plus des techniques de questionnement, $68 \%$ des enseignants ont cité la valorisation des élèves, $54 \%$, l'utilisation des connaissances antérieures et $45 \%$, la sensibilisation des parents à la nécessité de promouvoir le français au foyer comme stratégies pédagogiques.

\section{Perspectives des enseignants selon la recherche}

En plus d'explorer des stratégies pédagogiques pour appuyer les apprenants de la langue, nous avons aussi examiné le rôle des enseignants auprès des élèves dans leur apprentissage du français en milieu minoritaire. Dans ce contexte, les enseignants assument les rôles de modèles du français, de passeurs et de médiateurs culturels francophones (Laplante, 2001). Par le biais d'entrevues réalisées auprès d'enseignants d'écoles de langue française en Saskatchewan, LaPlante (2001) a montré à quel point les enseignants prenaient à cœur leur mission de passeurs de la langue et de la culture françaises. Selon LaPlante, les enseignants s'investissaient davantage parce qu'ils faisaient partie d'une école francophone. Ils se chargeaient de la responsabilité d'appuyer la francophonie de l'avenir.

Dans la même perspective, Gilbert, LeTouzé, Thériault et Landry (2004) ont mené un projet pour mieux comprendre le vécu de 672 enseignants d'écoles de langue française en milieu minoritaire. La recherche a démontré que ces enseignants manifestaient un engagement personnel au développement du français chez les élèves. En même temps, le sondage a confirmé les défis vécus par les enseignants dans ce contexte. Travaillant avec 
des élèves au bagage linguistique varié, les enseignants ont identifié le milieu anglodominant comme étant l'un de principaux défis. Pour répondre à ce défi, les enseignants ont recommandé l'intégration de cours de pédagogie en milieu minoritaire dans les programmes de formation et de perfectionnement professionnel et la production de ressources adaptées à ce milieu.

En outre, la FCE (2014a) a mené une étude pour mieux connaitre les expériences des enseignants de langue française en milieu minoritaire. Par le biais d'un questionnaire rempli par 815 enseignants d'écoles de langue française en contexte minoritaire, la FCE (2014a) a mis en relief les plus grands enjeux, dont les besoins diversifiés des élèves, l'importance de promouvoir le français dans un milieu anglodominant et le manque de formation appropriée. Elle a d'ailleurs recommandé la création d'un programme qui aiderait les enseignants à répondre aux besoins du milieu minoritaire et a insisté sur un appui accru de la part des gouvernements aux conseils scolaires pour aider les enseignants à promouvoir le français.

En ce qui concerne la francisation, Roy (2006) a relevé, par le biais d'entrevues menées auprès de 15 enseignants de français, que les enseignants ne se sentaient pas confiants dans leur capacité à répondre aux besoins des élèves ayant un bagage linguistique varié. Haché (2003), pour sa part, a dirigé des discussions auprès d'un groupe de 18 enseignants d'un conseil francophone en Ontario pour mieux connaitre leurs attitudes par rapport à la vitalité du français. Les enseignants ont reconnu que le français demeurait la langue parlée principalement pendant les cours. Ils ont avoué que, même en salle de classe, les élèves avaient de la difficulté à s'exprimer en français à cause d'un manque de vocabulaire. Pour faire face à ce défi, Haché a recommandé aux intervenants de mieux communiquer avec les parents anglophones, de leur offrir des cours de français et des services pour mieux les impliquer dans l'éducation de leurs enfants.

Gérin-Lajoie (2002), quant à lui, a reconnu l'importance primordiale accordée à la reproduction de la langue dans les écoles de langue française. Par le biais d'entretiens, Gérin-Lajoie a identifié la diversité de bagage linguistique des élèves comme étant l'un de principaux défis des enseignants, peu importe leur niveau d'expérience. Les 35 répondants aux entretiens de Gérin-Lajoie ont avoué qu'ils n'étaient pas bien préparés pour enseigner à un groupe d'élèves dont les compétences langagières étaient variées. D'autres recherches (p. ex. Brodeur, Deaulin et Bru, 2005 ; Cazabon, 2005 ; LeBlanc, 2009) ont soutenu également cette notion.

Dans leur étude, MacPhee, Turnbull, Gauthier, Cormier et Miller (2013) ont mené des entretiens auprès du personnel éducatif d'une école francophone à l'Île-du-PrinceÉdouard. Selon cette étude, le personnel se souciait de l'acquisition du français par les apprenants et, en même temps, se sentait motivé à pouvoir répondre à ce défi. Ce personnel a aussi établi le besoin de recherche dans le domaine de francisation. De même, cette étude a souligné l'importance de développer un programme de francisation qui permettrait de développer le vocabulaire et les habiletés littéraires des élèves.

En somme, les recherches ont indiqué que le bagage linguistique varié des élèves dans les écoles de langue française était un défi et que pour y faire face, les enseignants avaient besoin de perfectionnement professionnel. 


\section{Méthodes}

Afin d'appuyer le personnel enseignant du PAJE en contexte minoritaire, nous avons réalisé une recherche mixte. Nous avons d'abord interviewé les enseignantes et les éducatrices, observé leurs classes et participé à des réunions dans lesquelles nous avons parlé d'interventions. Nous avons ensuite interviewé les enseignantes et les éducatrices et observé les classes de nouveau pour comprendre les enjeux auxquels les enseignantes et les éducatrices doivent faire face et nous avons enfin tenté de leur offrir des stratégies pour y répondre. Pour récolter les données de cette étude, nous avons recouru à deux instruments, à savoir : des entrevues et un schéma d'observation. Nous avons également recouru à nos propres interventions pendant les réunions.

\section{Participantes}

Dans cette étude, il y avait 11 participantes, dont six enseignantes et cinq éducatrices. Toutes les enseignantes avaient obtenu leur qualification dans un programme de formation francophone au Canada pour l'enseignement du français langue maternelle. Elles avaient de 7 à 24 ans d'expérience en enseignement, puis de 5 à 13 ans d'ancienneté dans leur conseil scolaire. La majorité des éducatrices avaient été embauchées au début du PAJE et avaient travaillé dans des garderies avant de commencer à l'école. Elles avaient de 3 à 20 ans d'expérience en garderie.

\section{Instruments}

Pour récolter nos données, nous avons utilisé deux protocoles d'entrevue et un schéma d'observation.

Entrevues. Pour cette étude, nous avons mené deux entrevues avec les enseignantes et les éducatrices $(N=11)$. Le premier protocole d'entrevue a eu lieu à l'automne 2017 et le second, au printemps 2017. En automne, nous avons d'abord posé six questions démographiques au sujet des expériences des enseignantes et des éducatrices. Puis, nous avons posé quatre questions portant sur leurs expériences avec les apprenants de français.

Au printemps, nous avons posé les trois dernières questions de la première entrevue auxquelles nous avons ajouté quatre autres. Ces nouvelles questions portaient sur l'évaluation des interventions faites au cours de l'année par les enseignantes et les éducatrices pour répondre aux besoins des apprenants de français.

Schéma d'observation. Nous avons observé les classes en utilisant l'instrument d'observation « Adaptation de l'enseignement dans les classes de langue » (AECL) conçu par Arnett (2001). Arnett a créé l'AECL en adaptant l'outil Communicative Orientation of Language Teaching Observation Schema (COLT) de Spada et Frohlich (1995) pour décrire les classes de français langue seconde. Arnett y a ajouté des stratégies appropriées pour les adaptations faites par les enseignantes et les éducatrices dans le but de répondre aux besoins des élèves dans une classe de langue seconde.

À l'aide de cet instrument, nous avons établi les activités pédagogiques observées en classe en fonction du temps, exprimé en minutes, que l'enseignante leur avait consacré. Ces minutes constituent l'unité de mesure des observations. La grille nous a permis de 
noter les adaptations utilisées par les participantes en cochant l'adaptation associée à l'activité et en inscrivant la durée de l'activité en minutes. Par la suite, nous avons pu calculer le nombre total de minutes pendant lesquelles chaque descripteur a été observé et de calculer également le pourcentage représentant ce descripteur parmi l'ensemble des participantes en divisant le nombre de minutes d'observation. Les tableaux ci-dessous présentent les données combinées des 11 observations antérieures et ultérieures. Au total, nous avons complété 914 minutes d'observation avec une moyenne de 40 minutes par personne avant l'intervention et de 43 minutes après l'intervention.

\section{Interventions}

Pour répondre aux questions des enseignantes et des éducatrices soulevées au moment des entrevues et des observations, nous avons participé aux réunions de la communauté d'apprentissage du PAJE.

Réunions. Entre les entrevues de l'automne et celles du printemps, nous avons travaillé avec les enseignantes et éducatrices, ciblant les défis décrits au cours des entrevues et des observations, afin de mieux répondre aux besoins des apprenants de français. Avec les enseignantes et les éducatrices, nous avons participé à six réunions d'une demi-journée chacune. La première réunion s'est déroulée en novembre 2016. Nous y avons discuté de techniques de questionnement et du temps requis pour que les apprenants comprennent la question et puissent y répondre. Nous avons aussi travaillé avec une équipe d'enseignantes et d'éducatrices pendant leur temps de planification afin de leur offrir des pistes à suivre pour appuyer les progrès des apprenants de français. La réunion suivante a eu lieu en décembre. Nous y avons fait part des stratégies positives que nous avons observées en classe et discuté des étapes générales à suivre pour améliorer la compréhension du français des élèves (Annexe A).

En janvier 2017, la directrice de l'école a mené une discussion concernant l'usage de l'anglais en classe. Les enseignantes et les éducatrices se sont mises d'accord pour ne plus utiliser la méthode sandwich, et de ne plus utiliser l'anglais qu'en situation d'urgence ou de sécurité. Ainsi, nous avons décrit les stratégies gagnantes qui nous permettaient d'éviter, dans la mesure du possible, l'utilisation de l'anglais en classe et d'autres stratégies pour faire parler les élèves (Annexe B). En février et en avril, nous avons participé à la planification des enseignantes et des éducatrices.

\section{Résultats}

Dans cette section, nous présentons les résultats des entrevues préliminaires et des entrevues de suivi, et les données recueillies pendant les observations.

\section{Résultats des entrevues préliminaires}

Nous avons demandé aux enseignantes et aux éducatrices ce qu'elles faisaient pour répondre aux besoins linguistiques des apprenants de français dans leurs classes. Les participantes ont offert 17 stratégies différentes. Toutes les participantes ont établi la répétition du vocabulaire et des structures de phrase comme stratégie gagnante. Elles ont aussi indiqué que les aides visuelles étaient d'une importance primordiale. Plus précisément, elles ont mentionné les gestes, les images et l'usage des expressions de classe 
fixes. La moitié des participantes ont souligné l'utilité des chansons pour appuyer les apprenants de langue. Deux équipes ont aussi reconnu quatre autres stratégies, à savoir : l'usage des comptines, l'implication des parents, la routine et la méthode sandwich. En ce qui concerne la méthode sandwich, Alice a suggéré ceci :

$\mathrm{Tu}$ fais le sandwich, donc je fais français, anglais, français quand je vais dire des directives. Comme ça, je trouve que mes enfants qui sont purs anglophones, qui ont jamais entendu le français, c'est pas complètement chinois pour eux autres.

Quelques participantes ont aussi évoqué les stratégies suivantes : utiliser le ton de la voix et les émotions, parler seulement en français, utiliser les mots clés, encourager le français entre élèves, les faire parler en petits groupes et les motiver par l'usage d'un système de récompense.

Usage de l'anglais. À l'automne, nous n'avons pas posé de questions explicites au sujet de l'usage de l'anglais dans les classes, mais toutes les participantes ont mentionné cet usage. La majorité des équipes, soit $80 \%$ (4/5), ont utilisé l'anglais dans certaines situations. Par exemple, pour consoler un enfant et le mettre en confiance, Camille a recouru à l'anglais :

On essaie de ne pas utiliser l'anglais en salle de classe, mais parfois c'est incontournable; l'enfant devient frustré et tu vois qu'il ne comprend pas ce que tu lui dis. Donc, pas de choix que de lui parler en anglais. Mais en gros, je dois dire que ce n'est pas trop souvent qu'on doit parler en anglais.

Pour des raisons de sécurité, Laurence a dit :

C'est pas évident de parler en français en salle de classe quand ces élèves-là, comme l'élève autiste quand sa première langue est l'anglais, qui est en train de te mordre, c'est pas évident de dire «Ah ! Je vais lui parler en français » quand il comprend aucun mot en français. À un moment donné, tu veux protéger les autres élèves, il faut protéger les autres élèves ; donc, tu dois lui parler en anglais, mais on se fait dire qu'il ne faut pas parler en anglais.

De même pour recueillir des renseignements, Juliette a suggéré de recourir à l'anglais :

Puis, il y a des situations qu'on peut pas parler en français. L'élève, tu veux savoir s'il a mangé, tu y dirais 10 fois en français si il a mangé et tu sauras jamais ou une situation qui s'est passée, il était frappé et il a un bleu dans le visage, bien qu'est-ce que t'as eu, qu'est-ce que t'as eu, si je veux avoir de l'information de l'enfant pour sa sécurité bien là, je perds mon temps lui dire en français.

Et, Alice a proposé de parler à l'enfant en anglais pour mettre en place de nouvelles routines :

Parce que les premières semaines tu regardes qui comprend le français puis qui que c'est complètement l'anglais. Donc, à partir de ça c'est là. Mais quand je fais la 
routine du matin c'est tout en français. Puis les élèves sont déjà capables de suivre et c'est tout en français. Mais c'est quand je donne une directive d'accord «C'est le temps d'aller jouer », tu sais j'ai apporté des toutous en classe et tu dis «Quand tu prends un livre tu peux te prendre un toutou », mais là les élèves qui sont purs anglophones bien eux autres je sais qu'ils ont rien compris là je leur dis «Ceux qui veulent un toutou c'est correct, mais il faut que tu prennes un livre pour la lecture » là je vais répéter "If you want one of these teddy bears you have to take a book with it $»$. Puis après ça je le redis en français comme ça ils entendent plus le français, mais au début c'est ça tu dois faire-mon avis.

Quatre participantes ont indiqué qu'il y avait une progression continue selon laquelle elles avaient diminué l'usage de l'anglais avec les élèves dans leur propre pratique. Par exemple, selon Renée :

Au début, lorsque j'ai débuté, je les parlais seulement en anglais. D'après moi, c'était, il était pas pour comprendre ce que je demandais ; donc, je les parlais en anglais puis maintenant, je vois que en les expliquant et faisant des signes et en répétant que ça fait que ça vient à aider.

Puis, Juliette :

Oui. Bien moi, je disais, je parle français les trois quarts du temps. Puis, je fais le sandwich. Ça, c'était ma première année au PAJE. La deuxième année, j'ai dit bon, on va laisser faire le sandwich; on va parler le plus qu'on peut en français parce que là, répéter à toujours à l'anglais, je trouve que l'élève se fie qu'on va le dire en anglais. On va parler, à moins d'un cas d'urgence, okay là, on s'est aperçue que le cas d'urgence, c'était quand même un 10-20 pour cent de la journée. Alors, là, on l'a éliminé en fin d'année. L'année dernière, on dirait, à partir de mars, parce que là, à ce point, les élèves étaient pas mal bons ; on parlait toujours en français, à moins d'une situation où on voulait avoir une réponse de l'élève pour sa sécurité, on a recommencé comme ça cette année, ça va super bien.

\section{Résultats des entrevues de suivi}

Après la mise en œuvre des interventions, nous avons mené des entrevues de suivi avec les enseignantes et les éducatrices. Nous leur avons d'abord demandé d'évaluer l'utilité des interventions et la manière dont celles-ci ont influencé ou non leur pratique. Ensuite, nous leur avons demandé de nommer les stratégies qu'elles utilisaient pour faire parler les élèves en français entre eux et à quels moments elles choisissaient d'utiliser de l'anglais en salle de classe.

Évaluation des interventions. Nous avons demandé aux enseignantes et aux éducatrices d'évaluer l'utilité des interventions proposées au cours du projet pour appuyer les apprenants de français. La majorité $(n=8)$ des participantes ont apprécié les réunions.

Trois des participantes ont décrit l'influence des réunions sur leur usage de l'anglais en salle de classe. Par exemple, Renée avait ceci à dire : 
Moi, j’ai bien aimé les stratégies que tu nous as apportées. Les différentes façons d'incorporer la langue en salle de classe, ça j'aime ça. J'aimerais en avoir même plus souvent, juste pour nous donner des différentes idées. J'en ai une couple de petites mesdames qui, elles, parlent beaucoup l'anglais ; mais maintenant, je suis capable de leur parler en français et elles comprennent. Ceci a fait un gros changement et ça change aussi ma perspective de la langue, car je me trouvais toujours à utiliser l'excuse, " mais ils vont pas comprendre », et à cette heure, je me sens, comme si j'utilise plus de gestes, plus de différentes façons pour qu'ils puissent me comprendre, ils viennent qu'à comprendre qu'est-ce que tu veux. C'est toutes des petites stratégies et des idées que je trouve nous a aidées ensemble, en salle de classe beaucoup.

Il en était de même pour Charlotte qui a dit :

Je pense que les réunions qu'on a eues pour en parler, ceci m'a beaucoup aidée pour être plus consciencieuse de mon langage, que je dois vraiment parler plus en français. Car moi, j'accommodais trop. Puis, j'avais beaucoup d'anglais. Surtout au début, c'est normal, mais j'aurais dû . . A À tel point, il fallait arrêter. Puis, je savais vraiment que j'avais fait trop d'anglais quand un enfant avait dit « Hein, tu parles français? » puis, « Pourquoi tu parles en français ?». Puis, j'étais comme, c'est le français qu'on parle à l'école, en tout cas-Je vais être meilleure pour l'année prochaine, mais j'étais toujours honnête, que oui, j'utilisais l'anglais. Oui, et c'était l'enfant qui voulait se sentir sécure et qu'elle appartient, mais, je veux qu'ils comprennent, mais, je ne faisais pas de faveurs. Donc, les réunions ont peut-être apporté cela à la surface.

En considérant comment ces interventions ont influencé leur pratique, la majorité des enseignantes et des éducatrices ont mentionné que la mise en commun des stratégies était une expérience importante pour elles. La majorité des participantes $(n=8)$, dont Émilie et Charlotte, ont indiqué que les interventions avaient influencé leur usage de l'anglais. Par exemple, Émilie a déclaré ceci :

On s'est mis d'accord de . . quand utiliser l'anglais et là, on sait que ce n'est pas n'importe quand. Si l'on fait, tu peux essayer autant que possible de ne pas utiliser l'anglais, ils vont cheminer plus vite. Puis, comme je le vois . . . je pense que quand on parle moins l'anglais, les élèves se forcent plus à retenir les mots français, car ils savent «Ah! ok, je veux pas passer à travers de tout ça, les gestes, je vais m'en rappeler ».

Et Charlotte a dit :

Je suis plus consciencieuse de parler en français. Tu sais, de vraiment faire l'enfant répéter plus souvent, pas répondre trop vite, à cause, souvent, je répondais trop vite et peut-être en anglais. J'ai vraiment réalisé, c'est ça que je faisais. Puis, juste mettre plus l'emphase sur les gestes ... Et les enfants répondent très bien à ça . . . Après ça, les signes disparaissent et ils connaissent le mot. 
Stratégies pour faire parler français aux élèves entre eux. En répondant à la question concernant les stratégies qu'elles utilisaient pour faire parler français aux élèves entre eux, les enseignantes et les éducatrices ont parlé de six stratégies différentes, notamment : les récompenses $(n=3)$, les rappels $(n=2)$, les encouragements aux élèves à s'entraider avec les mots $(n=2)$, les jeux $(n=2)$, l'enseignement des structures dont ils avaient réellement besoin $(n=2)$ et les exercices de modélisation $(n=2)$. À ce sujet, les idées d'Émilie et de Charlotte semblaient représentatives de toutes les participantes. Émilie a dit :

Bien, je pense que, comme à ce temps ici dans l'année, on circule, on les fait penser, on les donne des rappels, on pourrait ajouter une récompense, des gommettes pour ceux qui parlent français. Cela est quelque chose que tous les enfants aiment beaucoup. Mais, je pense que c'est juste le fait qu'ils savent qu'on les écoute, on les entend, des fois, ils viennent à bout de dire quelques mots de français de se faire comprendre.

Pour sa part, Charlotte a déclaré ceci :

Des petits jeux qu'on peut faire, qu'on se parle en français aussi, ça aide. Juste, jouer dans un groupe, puis donner le mot en français pour que l'enfant soit capable, car l'enfant sait beaucoup, mais connait pas le mot, donc tu les vois, « dis le mot » et tu sais, on le remplace et on l'utilise souvent.

Quatre participantes ont mentionné que les élèves parlaient souvent anglais entre eux. Par exemple, Zoé a dit : « Mais, on a une classe d'anglophones donc . . . même les français parlent quand même en anglais avec leurs amis ». Et Renée a ajouté ceci :

Quand c'est les aires de jeu qui ne sont plus dirigées par l'adulte, ils vont nous parler avec les mots qu'ils connaissent en français. Mais, quand qu'ils sont avec les amis, c'est encore leur langue confortable qu'ils vont utiliser.

Nous avons demandé aux enseignantes et aux éducatrices si elles utilisaient de l'anglais avec les élèves et dans quelles circonstances elles faisaient régulièrement le choix de l'utiliser. Trois participantes n'utilisaient pas d'anglais dans la salle de classe. C'était le cas de Béatrice qui a affirmé, à ce sujet, que :

Utiliser l'anglais dans la classe avec les élèves, c'est un gros non-merci. Ç'a toujours été ma philosophie que, tu sais quoi, si on offre l'anglais, ils vont toujours se fier, mais si on simplifie et utilise plus de modelage visuel, même revenir aux chansons, ils vont l'apprendre beaucoup plus vite - beaucoup plus vite.

Les autres enseignantes et éducatrices ont donné des raisons qui justifiaient, selon elles, l'utilisation de l'anglais en salle de classe, entre autres et selon un ordre décroissant, le manque de compréhension, le temps de la journée (la fin de la journée) et la sécurité. Évoquant le manque de compréhension, Alice a mentionné ce qui suit : 
S'il y a un enfant qui est complètement perdu, et je n'ai aucune idée si elle a compris quelque chose, et si c'est quelque chose que je dois vraiment savoir, oui je vais la dire en anglais.

Dans la même perspective, Renée a dit :

Je sais qu'on n'est pas sensé d'utiliser la méthode sandwich, mais pour certains élèves, je me sens encore utiliser la méthode sandwich pour qu'ils puissent comprendre qu'est-ce que je veux dire.

Deux participantes ont décrit le défi posé par le manque d'aide-visuelles quand elles voulaient suivre une piste d'intérêt pour les élèves qui n'était pas prévue. Tel était le cas de Laurence qui a mentionné ceci : «J'ai encore de la difficulté avec les images, c'est le temps de trouver toutes les images qu'il faut. Surtout quand c'est quelque chose que tu n'as pas planifié ».

Croyances. Lors des entrevues du printemps, nous avons posé trois questions, reprises de l'automne, concernant les croyances. La majorité des participantes $(n=7)$ ont indiqué que leurs croyances au sujet de l'usage de l'anglais dans la salle de classe avaient changé à cause des expériences qu'elles avaient vécues pendant l'année. À ce sujet, Laurence a affirmé ceci :

On a toujours recours à anglais, c'est notre sécurité, mais on va lui dire en anglais puisqu'il ne comprend pas. Je réalise que « Ah! Il y a d'autres façons pour communiquer $\gg$.

De même, Charlotte a dit :

Je crois que oui, l'enfant peut apprendre le français plus facilement s'il s'immerge dans le français. Puis, j'ai même fait un petit sondage ou je connecte ceux qui sont anglais qui ont pris français à l'école, et j'ai demandé « C'est quoi qui t'a aidé à comprendre ? » et ils disent « Je ne comprenais rien, donc il fallait que j'apprenne et j'ai appris pour comprendre ». Donc, je dois parler en français toujours, car c'est ça, pour être capable de comprendre, on doit le parler.

Et Renée, d'ajouter ceci :

Moi, comme croyance je veux dire, j'étais un peu sceptique que les enfants pouvaient apprendre si vite et si facilement une autre langue sans toujours utiliser l'autre langue. Je veux dire, je ne croyais vraiment pas que rien fonctionnait autre que la méthode sandwich. J'aimais la méthode sandwich et je l'aime encore malheureusement. Là, j'essaye de m'en défaire.

Les participantes n'ont pas discuté de leurs croyances en lien avec l'emploi de l'anglais en salle de classe pendant les entrevues préliminaires. Elles n'y ont décrit que des influences positives sur leurs croyances, tandis que pendant les entrevues de suivi, une minorité parmi elles $(n=3)$ a aussi émis des doutes au sujet du bien-fondé de ne pas utiliser 
d'anglais en salle de classe. Par exemple, Léa a déclaré ceci : «C'est dur à répondre, c'est un petit peu décourageant, parce qu'on n'a pas d'appui à la maison. Puis, c'est un travail d'équipe, maison, école ». De même, Émilie a dit :

Il y a . . . avec certains élèves, je me pose la question encore . . . je me dis pourquoi le mettre en français, lui apprendre le français, je ne suis pas convaincue. Et avec nos petits [avec des défis autres que la langue] aussi, il semble qu'ils veulent parler en anglais. C'est leur premier choix de langue, alors je me dis, pourquoi pas aller avec cela?

\section{Résultats des observations}

Nous présentons certains résultats obtenus en comparant les observations faites avant les interventions et après les interventions. Dans cet article, nous nous limitons aux trois critères qui touchent à la langue, notamment : la langue parlée par l'enseignante ou l'éducatrice, l'usage de l'anglais et les mesures prises pour encourager l'usage du français. Nous décrivons d'abord les différentes classes avant de faire ces comparaisons et de présenter un sommaire de ces résultats.

Nous avons observé toutes les enseignantes et les éducatrices en salle de classe par deux fois. Nous avons utilisé un schéma par participante et par observation.

Description des classes. Nous avons noté toutes les activités en chronométrant le temps consacré à chacune d'elles afin de tenir compte de tous les descripteurs de classe et de toutes les adaptations suggérées dans le schéma AECL. Ce schéma nous a permis de fournir une description de chacune des classes.

Notamment, l'AECL offrait les moyens de suivre la langue parlée des enseignantes, des éducatrices et des élèves. Comme le démontre le Tableau 1 ci-dessous, personne n'a utilisé exclusivement l'anglais comme moyen de communication. En règle générale, les enseignantes et les éducatrices utilisaient le français, respectivement $60 \%$ du temps avant l'intervention et $72 \%$ du temps après l'intervention, ou un mélange de français et d'anglais, respectivement $40 \%$ du temps avant l'intervention et $11 \%$ du temps après l'intervention. Leur utilisation du français a augmenté de $12 \%$ après l'intervention, tandis que leur utilisation d'un mélange des deux langues a diminué de $29 \%$. Par ailleurs, après l'intervention, il y a eu des périodes où aucune communication n'était observée. Les élèves, quant à eux, ont utilisé le français $7 \%$ du temps avant l'intervention et $23 \%$ après l'intervention, et un mélange des langues $87 \%$ du temps avant et $60 \%$ du temps après l'intervention respectivement. 


\section{Tableau 1}

Langue parlée

\begin{tabular}{|c|c|c|c|c|}
\hline Descripteurs & $\begin{array}{l}\text { Minutes } \\
\text { observées } \\
\text { avant } \\
\text { l'intervention }\end{array}$ & $\begin{array}{l}\% \text { du temps } \\
\text { observé }\end{array}$ & $\begin{array}{l}\text { Minutes } \\
\text { observées } \\
\text { après } \\
\text { l'intervention }\end{array}$ & $\begin{array}{l}\% \text { du temps } \\
\text { observé }\end{array}$ \\
\hline $\begin{array}{l}\text { Enseignantes en } \\
\text { anglais }\end{array}$ & 0 & 0 & 0 & 0 \\
\hline $\begin{array}{l}\text { Enseignantes en } \\
\text { français }\end{array}$ & 267 & 60 & 337 & 72 \\
\hline $\begin{array}{l}\text { Enseignantes mélange } \\
\text { de langues }\end{array}$ & 117 & 40 & 50 & 11 \\
\hline $\begin{array}{l}\text { Absence de } \\
\text { communication des } \\
\text { enseignantes }\end{array}$ & 0 & 0 & 83 & 18 \\
\hline Élèves en anglais & 0 & 0 & 0 & 0 \\
\hline Élèves en français & 30 & 7 & 107 & 23 \\
\hline $\begin{array}{l}\text { Mélange de langues } \\
\text { élèves }\end{array}$ & 383 & 87 & 281 & 60 \\
\hline $\begin{array}{l}\text { Absence de } \\
\text { communication des } \\
\text { élèves }\end{array}$ & 31 & 7 & 82 & 17 \\
\hline
\end{tabular}

Les moyens permettant aux enseignantes et aux éducatrices de présenter le contenu de la leçon sont indiqués avant et après l'intervention dans le Tableau 2 ci-dessous. Les participantes ont utilisé l'anglais pour clarifier, respectivement $12 \%$ du temps avant l'intervention et $3 \%$ du temps après l'intervention, pour simplifier le langage utilisé, $2 \%$ du temps, et pour demander aux élèves de répéter, respectivement $7 \%$ du temps avant l'intervention et $3 \%$ du temps après l'intervention.

Nous avons constaté que les enseignantes et les éducatrices ont utilisé moins d'anglais et ont demandé moins aux élèves de répéter après l'intervention. Elles ont plutôt recouru à des concepts pour expliquer. 
Tableau 2

Présentation du contenu

\begin{tabular}{lllll}
\hline & $\begin{array}{l}\text { Minutes } \\
\text { observées } \\
\text { avant } \\
\text { l'intervention }\end{array}$ & $\begin{array}{l}\text { \% du temps } \\
\text { observé }\end{array}$ & $\begin{array}{l}\text { Minutes } \\
\text { observées } \\
\text { après } \\
\text { l'intervention }\end{array}$ & $\begin{array}{l}\text { \% du temps } \\
\text { observé }\end{array}$ \\
$\begin{array}{l}\text { Descripteurs } \\
\text { Expliquer les concepts }\end{array}$ & 0 & 0 & 15 & 3 \\
$\begin{array}{l}\text { Utiliser l'anglais pour } \\
\text { clarifier }\end{array}$ & 53 & 12 & 15 & 3 \\
$\begin{array}{l}\text { Utilisation de langue } \\
\text { plus simple }\end{array}$ & 8 & 2 & 8 & 2 \\
$\begin{array}{l}\text { Autre : l'élève répète } \\
\text { l'expression }\end{array}$ & 30 & 7 & 15 & 3 \\
\hline
\end{tabular}

La dernière catégorie du plan de schéma AECL offrait aux enseignantes et aux éducatrices des moyens de faire un suivi auprès des élèves (p. ex. renforcement positif), d'encourager certains comportements (incitatifs concrets), de donner de la rétroaction, d'encourager les élèves à utiliser le français et d'utiliser d'autres moyens pour encourager l'usage du français (signaux de renforcement).

Dans cette étude, selon les résultats du Tableau 3 ci-dessous, en ce qui trait aux mesures prises pour encourager l'usage du français, les participantes ont encouragé les élèves à utiliser le français comme moyen de communication, moins avant l'intervention, $10 \%$ du temps, qu'après, $35 \%$ du temps.

Tableau 3

Mesures prises pour encourager le français

\begin{tabular}{lllll}
\hline & $\begin{array}{l}\text { Minutes } \\
\text { observées } \\
\text { avant } \\
\text { l'intervention }\end{array}$ & $\begin{array}{l}\% \text { du temps } \\
\text { observé }\end{array}$ & $\begin{array}{l}\text { Minutes } \\
\text { observées } \\
\text { après } \\
\text { l'intervention }\end{array}$ & $\begin{array}{l}\% \text { du temps } \\
\text { observé }\end{array}$ \\
\hline $\begin{array}{l}\text { Descripteurs } \\
\begin{array}{l}\text { Encourager } \\
\text { l'utilisation du } \\
\text { français }\end{array}\end{array}$ & 43 & 10 & 166 & 35 \\
\hline
\end{tabular}

Comparaisons statistiques. Des tests de rangs signés de Wilcoxon ont été menés dans le but de comparer les variables observées avant et après nos interventions. Seulement deux variables étaient différentes de manière significative : l'utilisation du français par les élèves, et l'utilisation d'un mélange de français et d'anglais par les élèves.

Des résultats statistiquement significatifs ont été trouvés en comparant l'utilisation de la langue par les élèves avant et après nos interventions. En particulier, selon le Tableau 4 ci-dessous, le test des rangs signés de Wilcoxon a prouvé que l'utilisation du français était, d'un point de vue statistique, nettement en hausse après les interventions 
$z=-2,03, \mathrm{p}<, 04$ et que, parallèlement, leur utilisation d'un mélange d'anglais et de français diminuait de façon importante, $z=-1,96, \mathrm{p}<, 05$.

Tableau 4

Utilisation de la langue

\begin{tabular}{|c|c|c|c|c|}
\hline Descripteur & $\begin{array}{l}\text { Moyenne } \\
\text { avant le } \\
\text { classement }\end{array}$ & $\begin{array}{l}\text { Moyenne } \\
\text { après le } \\
\text { classement }\end{array}$ & $Z$ & $\begin{array}{l}\text { Valeur } \\
p\end{array}$ \\
\hline $\begin{array}{l}\text { Enseignante et enseignant } \\
\text { (l'utilisation de l'anglais) }\end{array}$ & 0,00 & 0,00 & 0,00 & 1,00 \\
\hline $\begin{array}{l}\text { Enseignante et enseignant } \\
\text { (l'utilisation du français) }\end{array}$ & 2,00 & 4,50 & $-0,41$ & ,69 \\
\hline $\begin{array}{l}\text { Mélange enseignantes et } \\
\text { éducatrices }\end{array}$ & 3,50 & 1,00 & $-1,76$ & ,08 \\
\hline Élève (l'utilisation de l'anglais) & 0,00 & 0,00 & 0,00 & 1,00 \\
\hline Élève (l'utilisation du français) & 2,00 & 4,33 & $*-2,03$ & ,04 \\
\hline Mélange élèves & 6,11 & 5,50 & $*-1,96$ & 05 \\
\hline
\end{tabular}

Discussion

Dans les entrevues préliminaires, les participantes ont nommé plusieurs stratégies qu'elles utilisaient pour appuyer les apprenants de langue dans leurs salles de classe. Elles ont également admis ressentir un malaise relatif à la question de l'utilisation de la langue anglaise en salle de classe. Il est à noter que les participantes ont parlé de ce malaise sans se faire poser de questions à ce sujet. Il est aussi significatif que la revue documentaire ne traitait pas de la question de l'usage de l'anglais dans une école de langue française. Ce manque de direction provenant de documents gouvernementaux et de recherches ainsi que l'absence de formation laissaient les enseignants à se débrouiller tout seul en ce qui concerne leur usage de l'anglais et son usage par leurs élèves.

Il se peut que les enseignantes et les éducatrices aient profité des réunions, en particulier de celles qui touchaient aux stratégies, à cause de l'absence de discussion à ce sujet. Elles ont indiqué que ces réunions avaient beaucoup influencé leur pratique et leurs croyances. De manière beaucoup plus précise, elles croyaient que les élèves pouvaient bien réussir sans recourir à l'anglais, ce qui a fait que la majorité d'entre elles ont diminué l'usage de cette langue en salle de classe. D'un côté, cette progression reflète la revue documentaire qui encourage une pédagogie de langue maternelle sans considérer la langue première des élèves, mais de l'autre, elle ne tient pas compte de la recherche et des approches du français langue seconde (p. ex. Cook, 2001) qui encouragent l'usage des langues premières des élèves en tant que ressource valable (p. ex. Cook 2008).

Les observations faites en classe étaient centrées sur l'usage des langues des enseignantes, des éducatrices et des élèves. Les participantes et les élèves ont augmenté 
leur utilisation du français et diminué leur utilisation d'un mélange d'anglais et de français après les interventions. La différence dans l'utilisation des langues de la part des élèves était statistiquement significative. Dans la mesure où cela s'appliquait à la présentation du contenu de la leçon, les enseignantes et les éducatrices utilisaient moins d'anglais pour clarifier des notions après les interventions. En plus, elles encourageaient les élèves à utiliser plus de français après les interventions qu'avant. Les observations ont également confirmé les données des entrevues, selon lesquelles, même si l'anglais était utilisé, une nouvelle attention était régulièrement portée à l'utilisation de la langue française en classe, ce qui avait comme résultat de réduire l'utilisation de l'anglais.

On pouvait ainsi anticiper une diminution de l'utilisation de l'anglais par les enseignantes et les éducatrices au cours de l'année scolaire. Par ailleurs, à cause des interventions effectuées pendant l'année, les participantes confirmaient avoir une plus grande conscience et une confiance accrue dans la capacité de leurs élèves d'apprendre le français sans utiliser l'anglais, ce qui les encourageait à réduire progressivement l'utilisation de l'anglais et les motivait aussi à inciter les élèves à utiliser davantage le français.

Compte tenu des contradictions observées entre l'utilisation de l'anglais par les enseignantes et les éducatrices et leur volonté de n'utiliser l'anglais qu'en cas d'urgence, l'emploi de l'anglais constitue un thème digne de discussions futures et d'autosurveillance. Selon la revue documentaire, l'objectif d'utiliser uniquement le français dans une école de langue française est lié au désir de se différencier des écoles d'immersion en reconnaissant le danger que pose l'anglais lorsqu'il devient dominant et fait obstacle à l'acquisition du français. En d'autres termes, l'objectif est de maintenir et de produire des citoyens dont la langue principale est le français. En conformité avec cet objectif, nous observons un recours presque exclusif aux stratégies du français langue maternelle, comme le veut la majorité des recherches et des documents de la revue documentaire, sans tenir compte de recherches récentes qui confirment l'importance de l'utilisation limitée et consciente des langues premières des élèves dans le but de renforcer l'acquisition d'une langue seconde (Cummins, 2007 ; Meiring et Norman 2002 ; Swain et Lapkin, 2000 ; Watanabe, 2008). L'utilisation des langues premières des élèves, en fait, n'est recommandée que pour traduire des mots complexes et abstraits ainsi que pour établir des routines en salle de classe.

Étant donné les expériences des participantes de cette recherche, il semble avantageux de continuer la discussion concernant l'utilisation de l'anglais en salle de classe, surtout dans le contexte d'écoles françaises en milieu minoritaire où la majorité des élèves sont souvent des apprenants de la langue. Cette discussion doit tenir compte non seulement des pratiques et des recherches quand à l'approche du français langue maternelle, mais devrait également se faire à la lumière des recherches en français langue seconde.

La correspondance devrait être adressée à Callie Mady.

Courriel : calliem@nipissingu.ca

\section{Références}

Arnett, K. (2001). The accommodation of grade 9 students with learning disabilities in the applied grade 9 core French classroom. Toronto, Canada: Ontario Institute for Studies in Education (OISE). 
Bell, D. (2007). Do teachers think that methods are dead? English Language Teaching (ELT) Journal, 61, 135-143.

Berger, M. J. (2003). Exploration du portfolio de l'enseignant comme outil de réflexion et de mise en oeuvre d'un curriculum. Revue suisse de l'éducation, 25(1), 125-142.

Berger, M. J. (2007). Interventions pédagogiques favorisant l'apprentissage de la lecture en contexte d'actualisation linguistique en français. Dans A. Dionne et M. Berger (dir.), Les littératies : perspectives linguistique, familiale et culturelle (p. 187-208). Ottawa, Canada : Presses de l'Université d'Ottawa.

Bours, P. et Lentz, F. (2009). Les littératies multiples : un cadre de référence pour penser l'intervention pédagogique en milieu francophone minoritaire. Cahiers francocanadiens de l'Ouest, 21, 127-150.

Brodeur, M., Deaudelin, C. et Bru, M. (2005). Le développement professionnel des enseignants. La Francophonie Dans Les Amériques, 154, 102-106.

Cazabon, B. (1997). L'enseignement en français langue maternelle en situations de minorité. Revue Des Sciences de L'éducation, XXIII(3), 483-508.

Cazabon, B. (2005). Pour un enseignement réussi du français langue maternelle: fondements et pratiques en didactique du français. Sudbury, Canada : Prise de parole.

Conseil des ministres de l'Éducation (CMEC). (2003). La francisation : parcours de formation. Projet pancanadien de français langue première à l'intention du personnel enseignant de la maternelle à la 2e année. Toronto, Canada : Conseil des Ministres de 1'Éducation. Repéré à http://204.225.6.243/else/francisation/cd-rom/

Conseil des ministres de l'Éducation (CMEC). (2004). Résultats pancanadiens des élèves francophones en milieu minoritaire au Programme d'indicateurs du rendement scolaire. Toronto, Canada : Conseil des Ministres de l'Éducation. Repéré à http://www.cmec.ca/Publications/Lists/Publications/Attachments/60/analysis.fr.pdf

Cook, V. (2001). Using the first language in the classroom. The Canadian Modern Language Review, 57(3), 402-423. doi:10.3138/cmlr.57.3.402

Cook, V. (2008). Second language learning and language teaching (4 $\left.{ }^{\text {th }} \mathrm{ed}.\right)$. London, United Kingdom: Hodder Education.

Cormier, M. (2005). La pédagogie en milieu minoritaire francophone : une recension des écrits Document établi par. Moncton, Canada : Fédération canadienne des enseignantes et des enseignants.

Cormier, M. (2011). La pédagogie en milieu francophone minoritaire. Dans J. Rocque (dir.), La direction d'école et le leadership pédagogique en milieu minoritaire (p. 287-306). Winnipeg, Canada : Presses universitaires de Saint Boniface.

Cormier, M. et Lowe, A. (2010). Étude des mesures d'accueil et d'accompagnement et de l'implantation de différents modèles d'intervention en francisation. Rapport de recherche. Moncton, Canada : Centre de recherche et de développement en éducation.

Cummins, J. (2007). Rethinking monolingual instructional strategies in multilingual classrooms. Canadian Journal of Applied Linguistics, 10(2), 221-240.

Fédération canadienne des enseignantes et des enseignants (FCE). (2014a). Enjeux de l'enseignement en contexte minoritaire francophone. Ottawa, Canada : Fédération canadienne des enseignantes et des enseignants.

Fédération canadienne des enseignantes et des enseignants (FCE). (2014b). Trajectoires de formation. Ottawa, Canada : Fédération canadienne des enseignantes et des enseignants. Repéré à http://www.pelf.ca/tf 
Gérin-Lajoie, D. (2002). Le rôle du personnel enseignant dans le processus de reproduction linguistique et culturelle en milieu scolaire francophone en Ontario. Revue des sciences de l'éducation, 28(1), 125-146. https://doi.org/10.7202/007152ar

Gilbert, A., Letouzé, S., Thériault, Y. et Landry, R. (2004). Le personnel enseignant face aux défis de l'enseignement en milieu minoritaire francophone. Ottawa, Canada : Fédération canadienne des enseignantes et des enseignants.

Gouvernement du Canada. (1969). La loi sur les langues officielles. Ottawa, Canada : Gouvernement du Canada.

Gouvernement du Canada. (1982). Loi Consitutionnelle de 1982. Ottawa, Canada : Gouvernement du Canada. Repéré à http://laws-lois.justice.gc.ca/fra/const/page15.html

Haché, D. (2003). Le profil ethnolinguistique d'un conseil scolaire franco-ontarien : projet d'aménagement linguistique. Dans H. Duchesne (dir.), Recherches en éducation francophone en milieu minoritaire : regards croisés sur une réalité mouvant (p. 5178). Winnipeg, Canada : Presses universitaires de Saint-Boniface.

Landry, R, (2003). Libérer le potentiel caché de l'exogamie. Moncton, Canada : Commission nationale des parents francophones.

Laplante, B. (2001). Enseigner en milieu minoritaire : histoires d'enseignantes œuvrant dans les écoles fransaskoises. Revue des sciences de l'éducation 27, 127-150. https://doi.org/10.7202/000311ar

LeBlanc, G. (2009). L'éducation en Acadie du Nouveau-Brunswick : une voie vers l'autosuffisance linguistique et culturelle. Fredericton, Canada : Commission sur l'école francophone.

Lentz, F. (2016). Travail pédagogique et construction identitaire en milieu francophone minoritaire : quelques observations à l'occasion d'un anniversaire. Cahiers francocanadiens de l'Ouest, 21(1-2) 317-386. https://doi.org/10.7202/1037178ar

MacPhee, M., Turnbull, M., Gauthier, R., Cormier, M. et Miller, T. (2013). Stakeholder consultation relating to non-francophone students enrolled in minority French language schools in PEI-parents, teachers and school leaders. Final report. Charlottetown, Canada : University of Prince Edward Island.

MacWillie, M. \& LeBlanc, M. (2010). La francisation au préscolaire : un modèle didactique. Dans D. Doyon et C. Fisher (dir.), Langage et pensée à la maternelle (p. 119-148). Québec, Canada : Presses de l'Université du Québec.

Maltais, C. (2007). Relation entre la littératie familiale en milieu francophone et l'incidence d'un programme de maternelle quatre ans à temps plein sur le développement du langage et de la lecture des enfants. Éducation francophone en milieu minoritaire, 2(1), 6-18.

Masny, D. (2003). Les littératies: un tournant dans la pensée et une façon_d'être», dans R. Allard (dir.), Actes du colloque pancanadien sur la recherche en éducation en milieu francophone minoritaire : bilan et prospectives (p. 157-165). Montréal, Canada : Association canadienne d'éducation de langue française.

Meiring, L. et N. Norman (2002). Back on target: Repositioning the status of target language in MFL teaching and learning. Language Learning Journal, 26, 27-35.

Ministère de l'éducation de l'Ontario. (2004). Politique de l'aménagement linguistique de l'Ontario. Toronto, Canada : Ministère de l'éducation de l'Ontario. Repéré à http://www.edu.gov.on.ca/fre/document/policy/linguistique/linguistique.pdf 
Ministère de l'éducation de l'Ontario. (2016). Programme de la maternelle et du jardin d'enfants. Toronto, Canada : Ministère de l'éducation de l'Ontario. Repéré à https://files.ontario.ca/books/eduthekindergartenprogramfrenchaodawebjuly28.pdf

Roy, S. (2006). Les enseignantes et les enseignants de français en contexte albertain : discours et représentations. Éducation et francophonie, 34(1), 177-192.

Spada, N., \& Frohlich, M. (1995). Communicative orientation of language teaching observation scheme: Coding conventions and applications. Sydney, Australia: National Centre for English Language Teaching and Research, Macquerrie University.

Swain, M., \& Lapkin, S. (2000). Task-based second language learning: The uses of the first language. Language Teaching Research, 4(3), 251-274.

Watanabe, Y. (2008). Peer-peer interaction between L2 learners of different proficiency levels: Their interactions and reflections. The Canadian Modern Language Review, 64(4), 605-635. 


\section{Annexe A}

\section{Des stratégies pour maximiser le français utlisé par l'enseignant}

Stratégies observées

- Dessiner au tableau

- Utiliser les gestes

- Écrire des mots au tableau

- Simplifier le vocabulaire

- Modifier le ton de voix

- Ajuster le débit
Autres stratégies possibles

- Utiliser les objets

- Répéter (différent de reformuler)

- Reformuler

- Verbaliser le vécu des élèves

- Encourager les élèves à s'entraider

- Faire partie des aires pour interagir

- Mimer 


\section{Annexe B}

\section{Des stratégies pour encourager les élèves à produire en français}

Stratégies observées

- Fournir des amorces des phrases

- Laisser les tirets pour l'élève à remplir

- Modeler

- Avoir les élèves modeler

- Donner le mot requis en français

- Offrir des choix

- Faire chanter les élèves

- Utiliser les mots précis comme le ptérodactyle au lieu du dinosaure

- Apprécier l'effort des élèves

- Allouer le temps nécessaire
Autres stratégies possibles

- Communiquer le même message de différentes façons

- Jeux de mots (les entendre, les voir)

- Utiliser les synonymes une fois un mot est établi

- Utiliser des phrases plus complexes une fois un énoncé simple est compris

- Encourager l'interaction entre élèves

- Fournir des textes audio

- Inviter les élèves à réitérer les chansons, parties des histoires, des comptines

- Les faire répéter, reformuler (balle ... je veux la balle) 